\title{
Adaptive Wideband Beamforming Based on Digital Delay Filter
}

\author{
Zeeshan Ahmad, Song Yaoliang, Qiang Du \\ School of Electronic Engineering \& Optoelectronic Technology, Nanjing University of Science \& Technology, \\ Nanjing (210094), Jiangsu province, P.R.China \\ engr.zeeshan@hotmail.com,ylsong@njust.edu.cn,qdu1023@gmail.com
}

\begin{abstract}
A novel adaptive wideband beamforming method is proposed, where beamforming is achieved by LMS based spacetime adaptive filtering algorithm. Conventional broadband beamforming requires desired signal to be incident from the broadside i.e. direction normal to the array. The new method overcome this constraint by make use of digital delay filters which compensate the delay of receiving data, so that the signal of interest can be treated as if it had arrived from the broadside. Then LMS based space-time adaptive filtering algorithm is applied to achieve beamforming. Another advantage of the proposed methodology is its low computational complexity while sustaining high resolution. The effectiveness and advantage of the proposed methodology is theoretically investigated, and computational complexity is also addressed. To verify the theoretical analysis, computer simulations are implemented and comparisons with other algorithms are made.
\end{abstract}

Index Terms - Wideband beamforming, Digital delay filter, LMS algorithm, Space-time adaptive filtering.

\section{INTRODUCTION}

The concept of beamforming is to steer the antenna beam in the direction of the desired signal, whilst suppressing signal from other directions [1]. Adaptive antennas that operate as purely spatial filters are also bandwidth limited, although not in the same sense as time-domain, frequency-domain or subspace processing approaches. For narrowband signals the time difference of signal arrival between antenna elements can be treated as a phase shift of the received signal. However, for wideband signals the difference in the array received signal complex envelope cannot be neglected. The bandwidth and multi-path limitations of purely spatial filters are often overcome with what is known as Space-Time Adaptive Processing (STAP) [2-5] or Space Frequency Adaptive Processing (SFAP) [2-4].

Conventional spacecraft control and communication are mainly narrowband communication systems, i.e. the maximum relative bandwidth of the space Unified S-band (USB) measurement and control system is 5\% [6-8]. The movement towards a globalized space communication and unprecedented explosion of space technology worldwide has opened a new window for broadband communication to develop newer methodologies in the field of spacecraft control and communication. It is this challenging environment to which broadband communication intend to contribute by 
evolving meaningful and optimal solutions to various problems of antenna arrays and related applications. Due to the low-received space communication signal power which makes the system vulnerable to failure [9], disruption and undesired interferences, beamforming based on spatial adaptive filtering is one the main technology to address and assess the possible failure modes and to develop strategies to detect such effects and correct them. Research on adaptive narrowband beamformers [10] have been gaining ground in the literature, but empirical studies on wideband beamforming remains relatively marginal and scarce. Wideband beamforming is the current direction of significant research in array signal processing.

The most classical approach of broadband beamforming is to use tapped delay-lines (TDLs) or FIR filter to accomplish beamforming in time-domain. This time domain broadband beamformer is equivalent to a time domain filter, which can form an independent frequency response to compensate the phase difference of the received signal with different frequency and the interference signal is suppressed by space-time filtering [11]. Frequency domain beamforming is another approach to deal with wideband signals. Frequency domain beamforming employing least mean square (LMS) algorithm is discussed in [12-15]. Another good approach proposed in [1] is to use sensor delay line. Comparison between SDL and TDL beamformers are presented in [16]. A new approach to wideband signal close to end-fire based on TDLs is discussed in [17]. In [18-20], the improved broadband beamforming algorithm proposed is under the assumption that the desired signal is incident on the array from the normal direction (broadside) i.e. $\theta=0^{\circ}$, then DMI (Direct Matrix Inversion) algorithm is applied to realize beamforming. To avoid such constraints and difficulties associated with conventional broadband beamforming, in this paper we propose a novel method of adaptive broadband beamforming based on LMS algorithm, which is suitable for signals impinges on array from an angle $\theta$ relative to the broadside. Some other advantages of this novel methodology include its low computational complexity, high resolution to steer the main lobe to the desired direction and suppress the broadband interferences to overcome the shortcomings of conventional algorithms. This anti-jamming technology can be used in the measurement and control of the space vehicles as well as the satellite communication applications.

The rest of this paper is organized as follows. Section 2 describes the basic wideband signal model, whereas Section 3 presents the structure of wideband beamforming based on digital delay filter. Section 4 covers the proposed Spatial-Temporal Adaptive Algorithm based on LMS and section 5 calculates the computational complexity of the proposed algorithm. To illustrate the validity and performance of the proposed algorithm, computer simulations are conducted, and the results are given in Section 6. Finally section 7 offers some conclusions drawn on the basis of simulation results. 


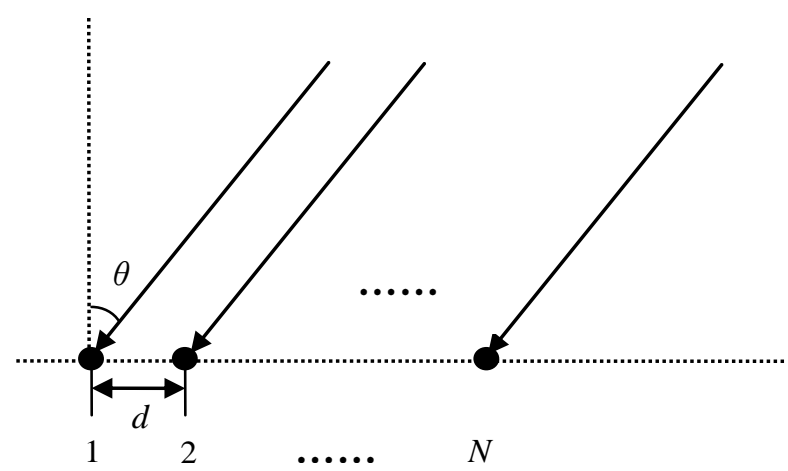

Fig. 1. Uniform linear array antenna structure

\section{SignAL MODEL}

Consider a uniform linear array with $N$ sensors as shown in figure 1 . The first array sensor located at the origin of the coordinates is assumed to be the reference sensor and $d$ is the spacing between two adjacent sensors. Consider the far-field signal model, assume that the desired signal and interference signal are of same frequency and non-coherent wideband signals, impinges on the array from an angle $\theta$ relative to the broadside, which refers to the direction normal to the array. The first array element receives the signal:

$$
x_{1}(t)=s(t)+\sum_{\mathrm{m}=1}^{\mathrm{M}} i_{m}(t)+n_{1}(t)
$$

Whereas, $i_{m}(t)$ is the $m$-th interfering signal $m=1,2, \cdots, M, n_{1}(t)$ is noise received on first array element, $s(t)$ is the desired signal received on first array element, a modulated signal can be expressed as:

$$
s(t)=m(t) \mathrm{e}^{\mathrm{j} 2 \pi f_{c} t}
$$

$m(t)$ is a baseband signal, $f_{\mathrm{c}}$ is the carrier frequency.

The propagation delay of the received signal from reference array element to the $n$-th array element can be expressed as:

$$
\tau_{n}=-(n-1) d \sin (\theta) / c
$$

Where, $n=1,2, \cdots, N, c$ is the speed of light, then the signal received on the $n-t h$ array element can be represented as:

$$
x_{n}(t)=s\left(t-\tau_{n}\right)+\sum_{\mathrm{m}=1}^{\mathrm{M}} i_{m}\left(t-\tau_{n}\right)+n_{n}(t)
$$

The received desired signal $s\left(t-\tau_{n}\right)$ is down-converted to baseband signal $m\left(t-\tau_{n}\right) \mathrm{e}^{-\mathrm{j} 2 \pi f_{c} \tau_{n}}$ before sampling.

The bandwidth $B$ of narrowband signal satisfy the condition of $B<<f_{c}$, and we can approximate $m\left(t-\tau_{n}\right) \approx m(t)$, so each array element receives the same signal envelope. For wideband signals, however, the above condition is not satisfied, so it is not the same as the narrowband signal uniform 
phase shift to compensate for the weight vector [21].

\section{WidEBAND BEAMFORMING BASED ON Digital Delay Filter StRUCtURE}

Compared with the conventional DMI wideband beamforming, the proposed beamformer employs LMS algorithm for calculating tap weight vector, which avoids computing the inverse of a matrix and also has smaller computational complexity. But such criterion has a limitation that it can only be used under the condition that the desired signal should impinge on the array from broad side i.e. $\theta=0^{\circ}$. So before calculating the weight vector, we need to compensate the delay of the antenna received signal via digital time delay filters in order to ensure that the desired signal impinges on the array from the direction normal to the array. Once the tap weight vector is obtained, then delay processing is applied to the optimum weight vector. Finally, we get the main lobe steered to the desired direction and nulls to the interferences.

The structure of digital time delay filter is shown in Figure 2. The core idea is the delay compensation $\tau_{n}$ in the time-domain, which makes the signal normal incident that is the angle of incidence $\theta$ is $0^{\circ}$. The Figure 2 shows that the structure of Digital time delay filter is composed of two parts, integer time delay filter and fractional delay filter.

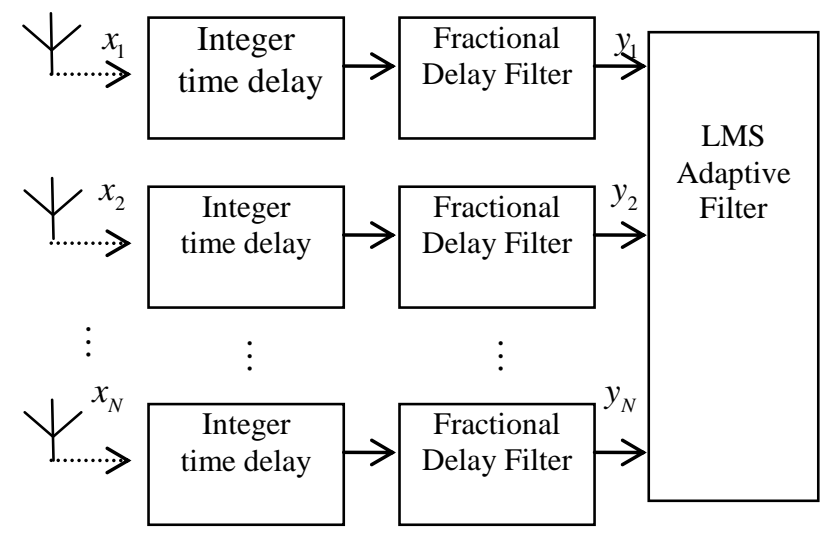

Fig. 2. Structure of Digital time-delay filter

Assuming the sampling period $T_{s}$ and the signal data received by the antenna after discretization, the $n-t h$ array element to compensate time delay can be expressed as:

$$
\frac{\tau_{n}}{T_{s}}=L+D
$$

So the total delay of any FD filter [22] can be split into an integer time delay of sampling interval $L=\operatorname{round}\left(\frac{\tau_{n}}{T_{s}}\right)$; and a fractional delay $D \in[-1 / 2,1 / 2)$. They can be calculated as:

$$
L=\operatorname{round}\left(\frac{\tau_{n}}{T_{s}}\right), D=\frac{\tau_{n}}{T_{s}}-L
$$

For the sampled data, the integer time delay of the sampling interval is first carried out, that is, to 
compensate the $L$, and then the fractional delay $D$ is compensated. Compensation $L$ can be achieved by delay of an integer multiple of the sampling interval $T_{\mathrm{s}}$ using digital delay line, having simpler hardware implementation. Compensation of $D$ can be realized by designing FIR filters. Because of its small value, the order of the filter required is not high, thereby reducing the hardware complexity.

The impulse response $h(n)$ of the FD FIR filter can be expressed as:[22]

$$
h(n)=\operatorname{sinc}(n-D)
$$

Clearly, for a non-integer $D, h(n)$ is infinite as well as non-causal and a filter with such an impulse response is thus non-realizable [22]. So we approximate the ideal filter by window method. The impulse response of the window function can be described as:

$$
h^{\prime}(n)=\mathrm{W}(n) \cdot \operatorname{sinc}(n-D), \quad 0 \leq n \leq N
$$

Window methods are extensively used in signal processing and its related applications. The most significant use of window can be found in design of digital filters, where a non-causal and infinite ideal impulse response is converted to a finite impulse response (FIR) filter design [23]. Though, the window method is numerical efficient in the sense that the ideal response is simply multiplied by a window [24]. However, limitations imposed on this approach to choose the optimal window method is quite complicated.

Taking into consideration advantages and disadvantages of available window methods [22], we have chosen Chebyshev window characterized by a minimum main-lobe width for a given side-lobe attenuation [23]. Chebyshev Window has the unique property that all its side-lobes are equal and the side-lobe height is the same at all frequencies [25]. This effect is shown in Figure 3 below.

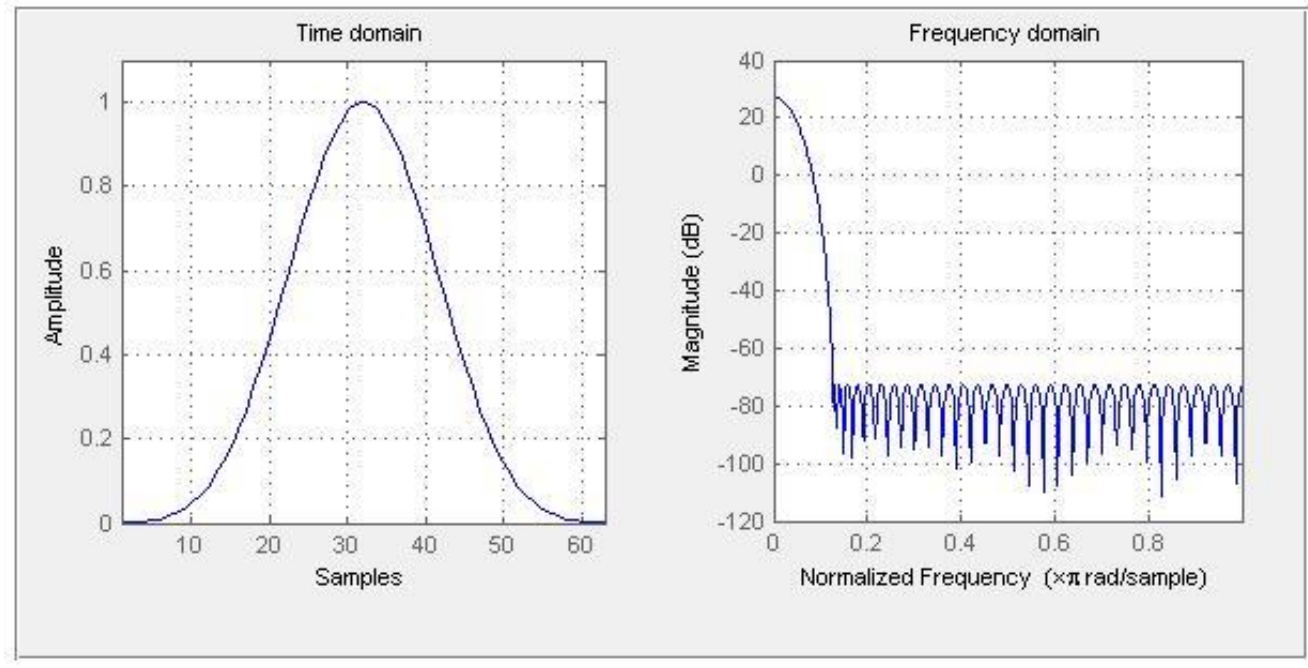

Fig. 3. Default length $\mathrm{N}=64$ Dolph-Chebyshev window with $100 \mathrm{~dB}$ relative sidelobe attenuation

The Dolph-Chebyshev Window function $w_{0}(n)$ in discrete time domain can be represented by the following equation [26-27] 


$$
w_{0}(n)_{D / C h}=\frac{1}{N} \sum_{k=0}^{N-1} W_{0}(k) e^{i 2 \pi n k / N}
$$

$W_{0}(k)$ is the Fourier coefficient and can be derived as:

$$
W_{0}(k)_{D / C h}=(-1)^{k} \frac{\cos \left\{N \cos ^{-1}[\beta \cos (\pi k / N)]\right\}}{\cosh \left[N \cosh ^{-1}(\beta)\right]}
$$

Where $\beta$ is a fixed value parameter described by the following equation:

$$
\beta_{D / C h}=\cosh \left[\frac{1}{N} \cosh ^{-1}\left(10^{\alpha}\right)\right]
$$

And

$$
\alpha=\cosh \left[\frac{1}{N} \cosh ^{-1}\left(10^{\frac{A}{-20}}\right)\right]
$$

The width of the main-lobe and the resulting filter transition-band can be controlled by varying $N$. The ripple ratio (Side-lobe level) can be controlled by parameter ${ }^{\alpha}$.

\section{SPATIAL-TEMPORAL ADAPTIVE ALGORITHM BASED ON LMS}

Space-Time Adaptive technology can transform a one-dimensional spatial filtering into twodimensional of time and space, forming a model of a two-dimensional space-time processing. After the process of digital delay line and fractional time delay filtering for the sample data, it will turn into the structure of space-time adaptive filtering based tap delay lines, as shown in Figure 4. In its discrete form, the TDL system is replaced by a finite impulse response (FIR) filter and adaptive algorithms can be realized by digital circuits [28]. It will realize the beamforming by adjusting the order of FIR filters/TDLs and the weight of the taps. The order of the TDLs is decided by the bandwidth of the impinging signals [29]. Generally, higher the bandwidth, the longer the TDLs [30].

As shown in Figure 4, there are $N$ array elements in the structure and each channel is connected to $J$ order FIR filter, and the output is as follows [31]:

$$
z(t)=\boldsymbol{w}^{\mathrm{H}} \boldsymbol{y}(t)
$$

Where; $\boldsymbol{w}$ is two dimension weight vector in space-time and holds all the $N J$ sensor coefficients, its size is $N J \times 1$ expressed as follows

$$
\boldsymbol{w}=\left[\begin{array}{llll}
\boldsymbol{w}_{1} & \boldsymbol{w}_{2} & \cdots & \boldsymbol{w}_{J}
\end{array}\right]^{T}
$$

Whereas, for the $j-t h$ column vector $\boldsymbol{w}_{j}, j=1,2, \cdots, J$ whose size is $N \times 1$, contains the $N$ complex conjugate coefficients found at the $j-t h$ tap position of the $N$ TDLs, and is expressed as:

$$
\boldsymbol{w}_{j}=\left[\begin{array}{llll}
w_{1, j} & w_{2, j} & \cdots & w_{N, j}
\end{array}\right]^{\mathrm{T}}
$$

And $\boldsymbol{y}(t)$ is the input data of dimension $N J \times 1$ obtained after the delay compensation of $N \times 1$ dimensional data through TDLs structure. With $y_{n j}(n=1,2, \cdots, N ; j=1,2, \cdots . J)$ denote the $n-t h$ 
channel to the $j-t h$ data after the delayed output, the input data vector can be expressed as:

$$
\boldsymbol{y}=\left[\begin{array}{llll}
\boldsymbol{y}_{1}(t) & \boldsymbol{y}_{2}\left(t-T_{s}\right) & \cdots & \boldsymbol{y}_{J}\left(t-J T_{s}\right)
\end{array}\right]^{\mathrm{T}}
$$

Where the $j$-th column vector $\boldsymbol{y}_{j}\left(t-j T_{s}\right)$ is the input data of dimension $N \times 1$, expressed as:

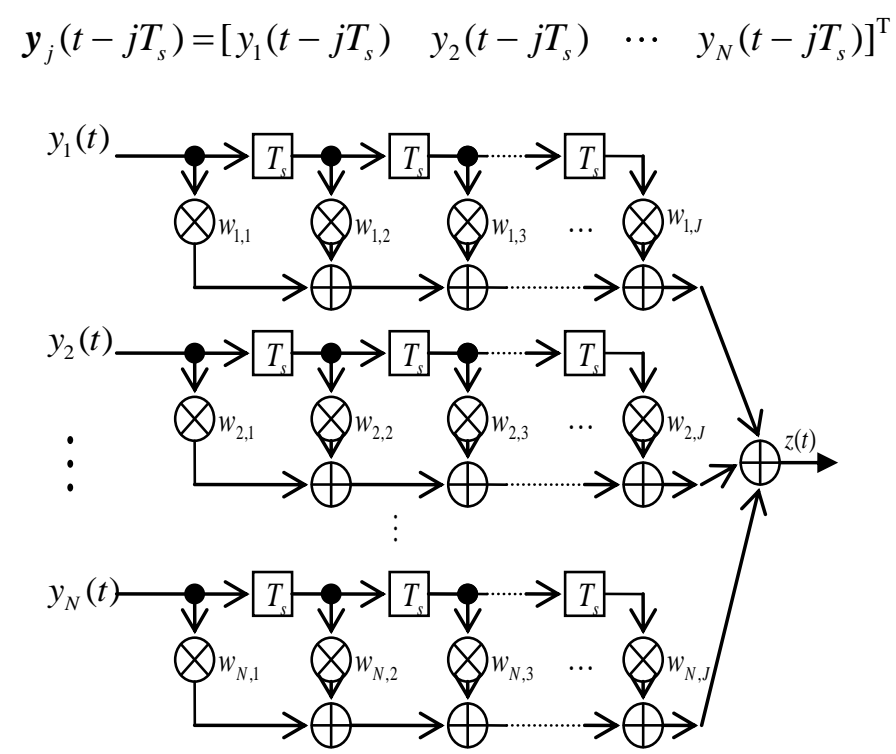

Fig. 4. Space-time adaptive beamforming based on the TDLs

The weight vector of the taps can be obtained through a variety of algorithms when the desired signal is incident from the normal direction. According to the DMI realization principle, the optimal solution solved by the Lagrange multiplier method has relation to the inverse of the correlation matrix $\boldsymbol{R}_{y y}^{-1}$ of the inputs, and can be expressed as follows:

$$
\boldsymbol{w}_{\mathrm{opt}}=\boldsymbol{R}_{y y}^{-1} \boldsymbol{C}\left(\boldsymbol{C}^{\mathrm{H}} \boldsymbol{R}_{y y}^{-1} \mathrm{C}\right)^{-1} \boldsymbol{f}
$$

The iterative method of LMS can be derived with space-time adaptive filter and Frost LMS algorithm in spatial filtering [31].

There are $J$ constraints in the space-time structure of $J$-order filters, and the constraint equation is as follow:

$$
\boldsymbol{C}^{\mathrm{H}} \boldsymbol{w}=\boldsymbol{f}
$$

Where the $N J \times J$ dimensional matrix $C$ is termed as the constraint matrix, and $f$ is the $J \times 1$ gain (constant) vector or $J$ being the number of constraints. The iterative equation obtained by Frost LMS algorithm is as follow:

$$
\boldsymbol{w}[k+1]=\boldsymbol{w}[k]-\mu\left(\boldsymbol{R}_{y y} \boldsymbol{w}[k]+\boldsymbol{C} \lambda[k]\right)
$$

where $\mu$ is the iterative step size, $\lambda$ is the Lagrange multiplier. $\boldsymbol{w}[k+1]$ must satisfy the constraint in equation (19), so we substitute equation (20) into equation (19) to get $\lambda[k]$. Then we substitute into $\lambda[k]$ the iteration equation in (20) and arrive at:

$$
\boldsymbol{w}[k+1]=\boldsymbol{C}\left(\boldsymbol{C}^{\mathrm{H}} \boldsymbol{C}\right)^{-1} \boldsymbol{f}+\boldsymbol{P}\left(\boldsymbol{w}[k]-\mu \boldsymbol{R}_{y y} \boldsymbol{w}[k]\right)
$$


where

$$
\boldsymbol{P}=\boldsymbol{I}-\boldsymbol{C}\left(\boldsymbol{C}^{\mathrm{H}} \boldsymbol{C}\right)^{-1} \boldsymbol{C}^{\mathrm{H}}
$$

The initial weight is

$$
\boldsymbol{w}[0]=\boldsymbol{C}\left(\boldsymbol{C}^{\mathrm{H}} \boldsymbol{C}\right)^{-1} \boldsymbol{f}
$$

The better approximate value of the correlation matrix as a time-average can be computed as:

$$
\tilde{\boldsymbol{R}}_{y y}=\frac{1}{K} \sum_{\mathrm{k}=1}^{\mathrm{K}} y(k) y^{\mathrm{H}}(k)
$$

$K$ is the number of snapshots, and then using the simple approximation of correlation matrix $\tilde{\boldsymbol{R}}_{y y}$ to replace $\boldsymbol{R}_{y y}$, the final weight vector can be calculated by following iterative equation as:

$$
\boldsymbol{w}[k+1]=\boldsymbol{C}\left(\boldsymbol{C}^{\mathrm{H}} \boldsymbol{C}\right)^{-1} \boldsymbol{f}+\boldsymbol{P}\left(\boldsymbol{w}[k]-\mu e^{*}[k] y[k]\right)
$$

\section{COMPutational PeRformance Analysis}

The computational complexity of the DMI algorithm is compared with that of the proposed LMS algorithm. The comparison is based on a count of the total number of complex multiplications and complex additions involved in each of these two algorithms as an indication of computational complexity of adaptive algorithms. This provides a reasonably accurate basis for comparing the computational complexity of these two algorithms.

Unlike the new algorithm, the conventional DMI algorithm must seek $\boldsymbol{R}_{y y}$, and subject to a matrix inversion operation to solve the optimal weight vector. The weight vector is calculated according to equation (18) which is computationally intensive. In practice, the covariance matrix is estimated by a finite number $K$ of time domain samples (snapshots). In the simulations, we used the forwardsmoothing algorithm to estimate the equation (24).

The new algorithm is based on equation (25) using the iterative calculations to get the optimal value, without knowing the true second order statistics information of the signal data, and $\boldsymbol{C}\left(\boldsymbol{C}^{\mathrm{H}} \boldsymbol{C}\right)^{-1} \boldsymbol{f}$ and $\boldsymbol{P}$ are fixed matrix under known array conditions, need to be calculated only once. It should be noted that the computation of weights by proposed method computes $K$ iterations compared to matrix inversion required by the DMI algorithm and thus the proposed method is computationally efficient.

In the structural model based on a tapped delay line, $\boldsymbol{R}_{\mathrm{yy}}$ is the covariance matrix of $N J \times N J$ dimension. According to the conventional method of matrix inversion, any element of the inverse matrix $\boldsymbol{R}_{y y}^{-1}$ is the division of the $N J-1$ matrix determinant by $\boldsymbol{R}_{y y}$ matrix determinant. The matrix inversion requires $(N J)^{4}-3(N J)^{3}+4(N J)^{2}-N J \quad$ complex multiplications and $(N J)^{3}-2(N J)^{2}+N J-1$ complex additions, so only the computation of inverse covariance $W(N, J)$ is 


$$
W(N, J)=(N J)^{4}-2(N J)^{3}+2(N J)^{2}-1
$$

From equation (26), it can be seen that the magnitude of computational cost in computing the inverse covariance $\boldsymbol{R}_{y y}^{-1}$ has reached to $(N J)^{4}$. The value of $\boldsymbol{R}_{y y}$ is related to the value of $K$. The higher the value of $K$, the more closer the estimated $\boldsymbol{R}_{y y}$ is to the true covariance matrix, so the value of $K$ is not too small. The size of $W(N, J)$, number of array elements $N$ and filter order $J$, are related to the number of snapshots $K$ and its value is large.

The optimal weight vector of DMI algorithm and the proposed algorithm is calculated by complex multiplication and addition operations, in accordance with equation (18) and (25) respectively. The total amount of computations $W 1$ required by the DMI algorithm is as follows:

$$
\begin{gathered}
W 1=\left(N^{4}+1\right) J^{4}+\left(-2 N^{3}+6 N^{2}+2 N-2\right) J^{3}+ \\
{\left[(2 K+2) N^{2}-2 N+2\right] J^{2}-N J-1}
\end{gathered}
$$

The total amount of computations $W 2$ of the new algorithm is as follows:

$$
\begin{aligned}
W 2= & 2 J^{4}+\left(2 N^{2}+8 N-4\right) J^{3}+ \\
& {\left[2 N^{2} K+2\right] J^{2}+5 N K J-K-2 }
\end{aligned}
$$

Comparing equation (27) and (28), we find that the computational complexity of $W 1$ is much higher than $W 2$, so the proposed algorithm not only reduces the computational complexity, but also easy to implement in real-time engineering applications.

\section{Simulation Results}

In this section, the proposed beamformer is evaluated by computer simulations. A uniform linear array composed of 8 elements with 7-order tap delay of digital filter is simulated in MATLAB. Elements are assumed to be omni-directional, and spacing between the adjacent array elements is $d=\lambda_{\min } / 2$. Assuming the space has two signals, desired signal comes from the look direction $\theta_{1}=20^{\circ}$ and the wideband interferences is incident on the array at $\theta_{2}=-20^{\circ}$. The sampling frequency is $f_{s}=400 \mathrm{MHz}$.

In high SNR simulation environment, we use a center frequency of $f_{c}=100 \mathrm{MHz}$, the modulating signal bandwidth (B) of $50 \mathrm{MHz}$, interference signal is non-coherent wideband signal of the same frequency and signal bandwidth and the noise is random white noise, obeying the normal distribution law. After receiving data through digital delay filter, DMI algorithm and proposed LMS algorithm are used to realize the broadband signal beamforming.

Figure 5 is the 3-D beam pattern of DMI algorithm. $x$-axis is the angle of arrival, the search range $\left[-90^{\circ}, 90^{\circ}\right], y$-axis is the normalized frequency $f \in[0,1]$, corresponds to the frequency range $\left[0, f_{s} / 2\right]$. The $50 \mathrm{MHz}$ desired signal corresponds to the frequency range $[75,125] \mathrm{MHz}$, i.e. $y$-axis range interval of $[0.375,0.625]$. From Figure 5, it can be observed that the main lobe is pointing in the 
$\theta=20^{\circ}$ direction. The most deep nulls are in the range of $y \in[0.3,0.7]$, and the null in the interference direction $\theta=-20^{\circ}$ significantly suppresses the wideband interference.

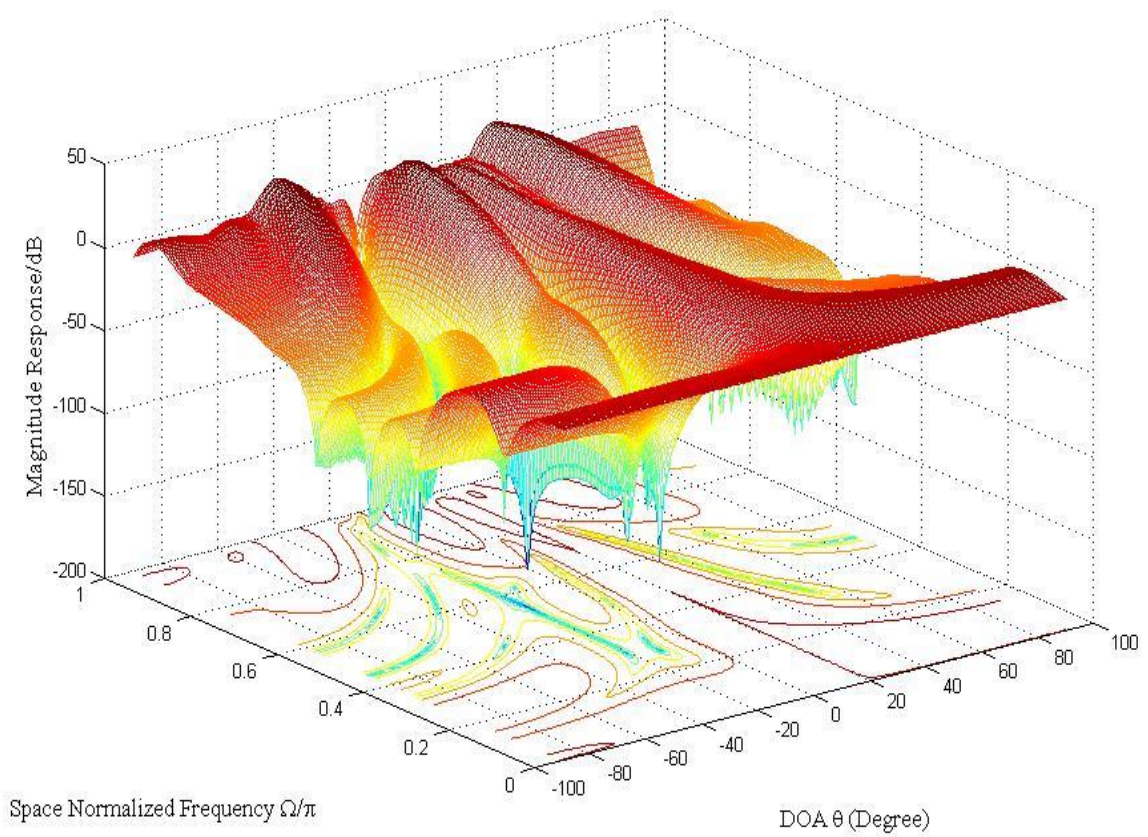

Fig. 5. A 3-D wideband Beam Pattern of DMI algorithm

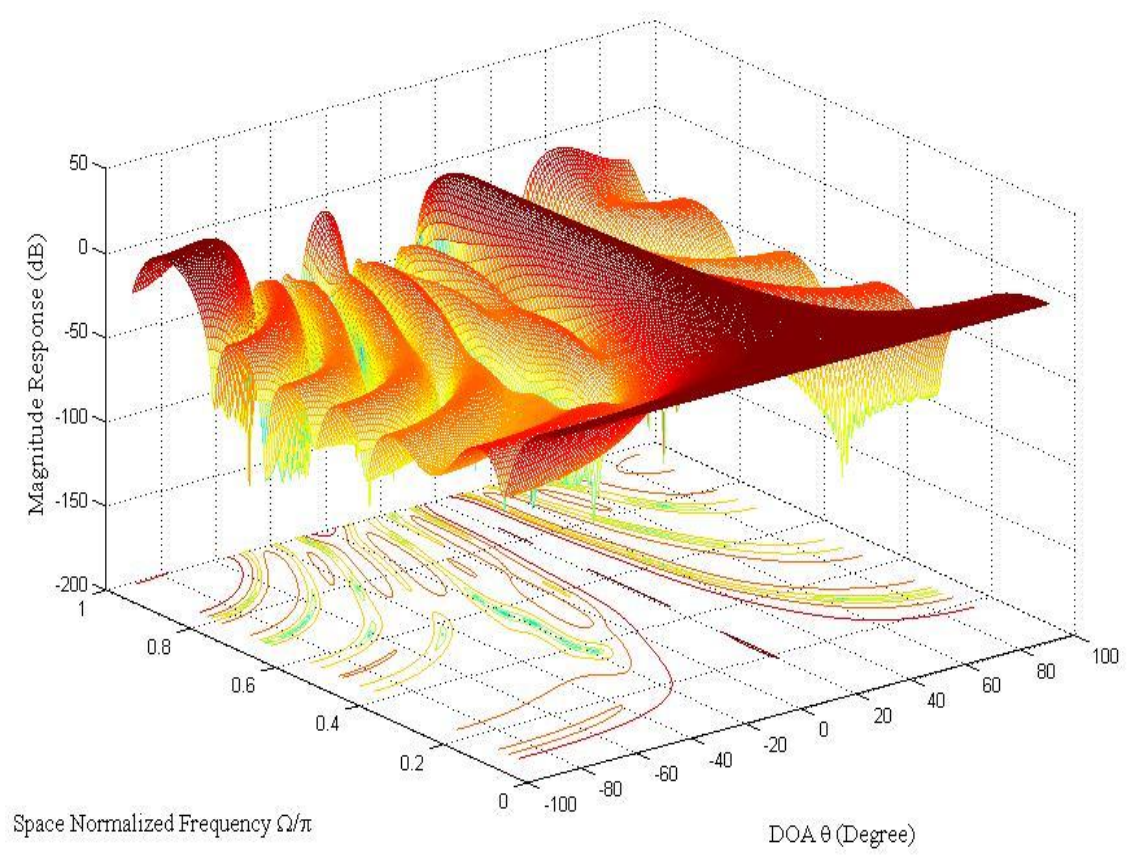

Fig. 6. A 3-D wideband Beam Pattern of Proposed algorithm

Figure 6 is the 3-D beam pattern under the proposed methodology. From the figure it can be observed that the main lobe is steered to the desired signal direction that is $\theta=20^{\circ}$. In the frequency 
range $y \in[0.35,0.68]$, the deepest null in the direction $\theta=-20^{\circ}$, effectively suppressed the broadband interference. This indicates the successful wideband beamforming operation by LMS algorithm based on digital delay filter.

Like DMI algorithm, the new methodology is also high resolution to steer the main beam in the desired signal direction and place deeper nulls in the interferer's direction. So, the performance the proposed beamformer is comparable with that of the conventional DMI beamformer.

It should be noted that if the desired signal is incident from the direction other than broadside, the conventional wideband beamformer without the digital delay filter or pre-steered delays is unable to do beamforming. This effect is shown in the Figure 7 below. The desired signal comes from the look direction $\theta_{1}=20^{\circ}$ and the wideband interferences is incident on the array at $\theta_{2}=-20^{\circ}$. The conventional wideband beamformer without delay filter forms the main beam at 0 degrees, while the proposed methodology employing digital delay filter is able to steer the main beam in the direction of desired signal (20 degrees).

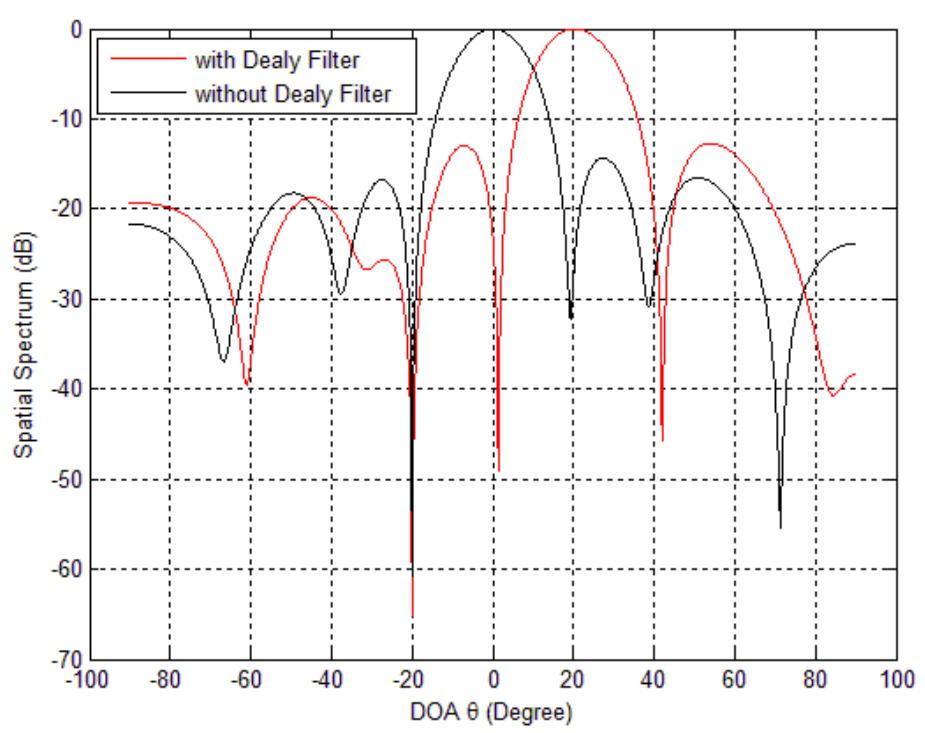

Fig. 7. The comparison of between the conventional beamformer and proposed beamformer for desired signal incident from the non-broadside direction.

To observe the computation performance of the algorithms, equation (23), and (24) shows that the amount of computations of the DMI algorithm and proposed algorithm are related with the values of $N, J$ and $K$. By varying these three variables, the computational complexity of both the algorithms are compared.

The computational complexity of DMI algorithm and proposed algorithm are plotted in Figure 8 with fixed number of tapped delay-lines $J=7, y$-axis represents the amount of computations i.e. computational complexity and $x$-axis represents the number of sensors. 


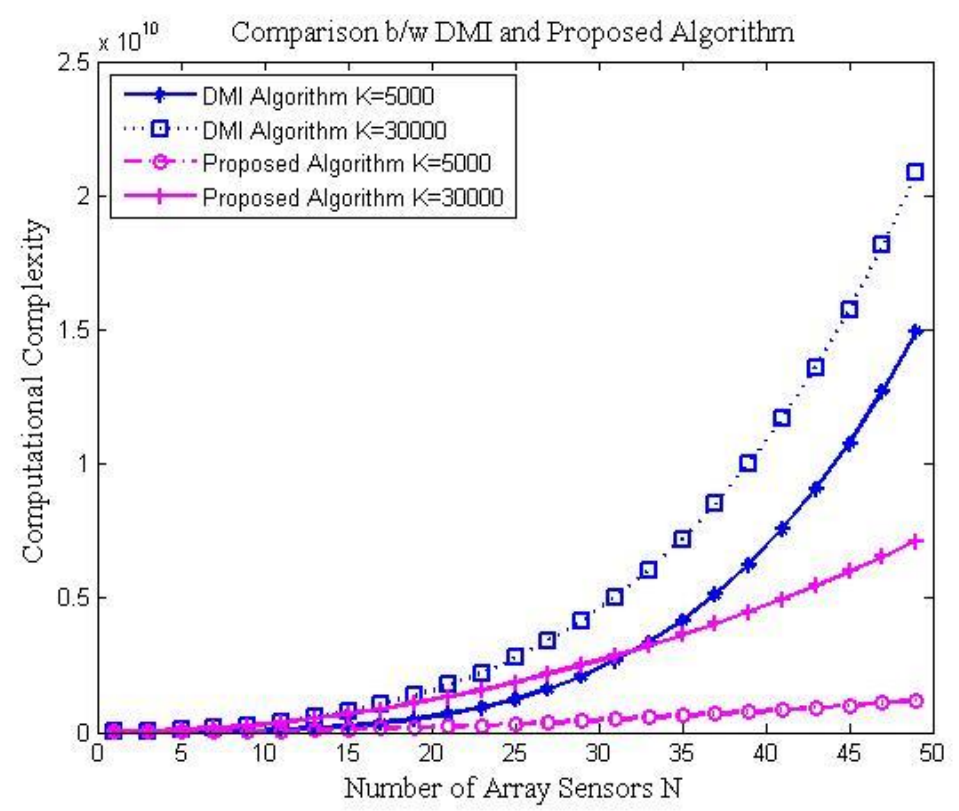

Fig. 8. The comparison of computational complexity between the proposed algorithm and the DMI algorithm under a fixed $\mathbf{J}$

As can be seen from the figure above, the computational complexity of both the algorithms increases with the increasing values of $N$ and $K$. So an increasing trend is observed in the complexity of both the DMI and proposed algorithm under fixed number of taps and with the increasing number of sensors and snapshots. Under the same value of $K$, the new algorithm is relatively less computational complex than DMI algorithm. Even, when $N>33$, the proposed algorithm with $K=30000$ is less computational complex than the DMI algorithm with $K=5000$. Clearly, the proposed algorithm significantly reduces the computational complexity as compared to DMI algorithm, for higher values of $N$.

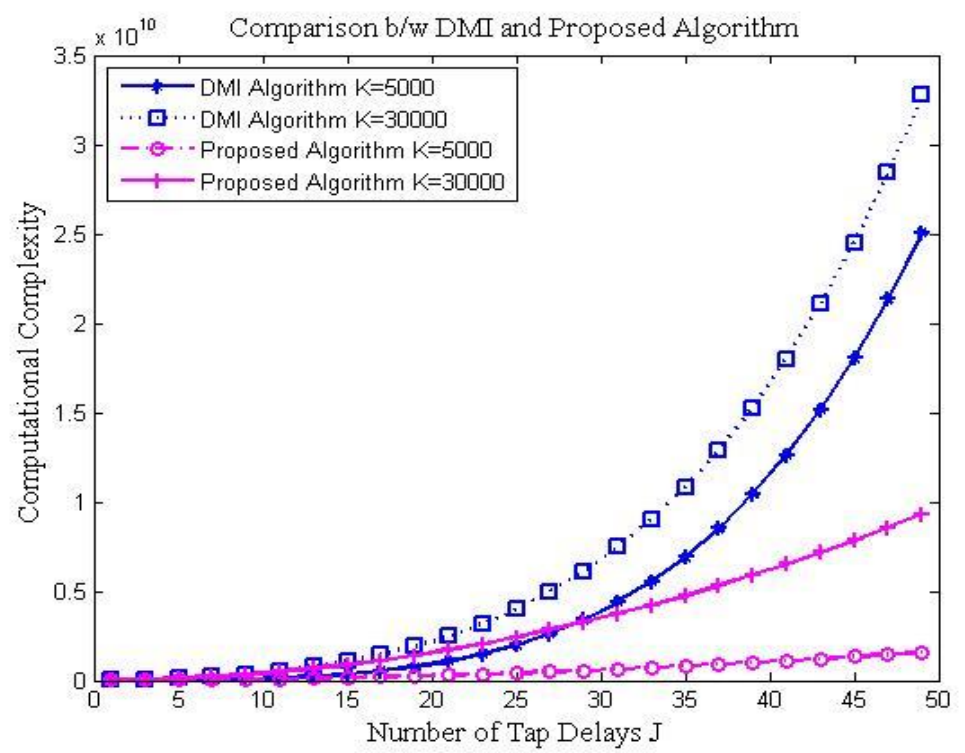

Fig. 9. The comparison of computational complexity between the proposed algorithm and the DMI algorithm under a fixed $\mathrm{N}$ 
The computational complexity of DMI algorithm and proposed algorithm are plotted in Figure 9 with fixed number of array elements $N=20$. The computational complexity of both the algorithms show an increasing trend with the increasing values of $J$ and $K$. The comparison of the proposed algorithm and DMI algorithm under the same value of $K$ shows that the new algorithm is relatively less computational complex. For $J>29$, the proposed algorithm with $K=30000$ is less computational complex than DMI algorithm with $K=5000$. Clearly, for higher values of $J$, the proposed algorithm significantly reduces the computational complexity as compared to DMI algorithm.

\section{CONCLUSION}

In this paper, we have proposed a new adaptive wideband beamforming methodology. The proposed methodology employ LMS algorithm based on the formation of a digital delay filter. The concept behind this new methodology is to overcome the constraint that signal of interest should be impinged on the array from the broadside. Simulations have demonstrated the superiority and validity of the proposed methodology. The appealing advantage of the proposed methodology lies in that it not only achieves the broadband interference suppression, high resolution to steer the main beam in the direction of desired signal and null the interferences, but is also less computational complex by reducing the amount of computations to be easily realized in practical systems.

\section{ACKNOWLEDGMENT}

The authors gracefully acknowledge the support of the National Natural Science Foundation (NSFC) Project (61271331) and project (61071145) of China.

\section{REFERENCES}

[1] Liu W. Adaptive wideband beamforming with sensor delay-lines. Signal Processing, 2009, 89(5): 876-882.

[2] R. L. Fante, J. J. Vaccaro. Wideband Cancellation of interference in a GPS Receive Array. IEEE Transactions on Aerospace and Electronic Systems, 2000, 36(2): 549-564.

[3] W. L. Myrick, M. D. Zoltowski, J. S. Goldstein. Anti-jam space-time preprocessor for GPS based on multistage nested Weiner filter. Military Communications Conference Proceedings, 1999, 1: 675-681.

[4] R. L. Fante, J. J. Vaccaro. Cancellation of Jammers and jammer multipath in a GPS receiver. IEEE Aerospace and Electronic Systems Magazine, 1998, 13(11): 25-28.

[5] G. F. Hatke. Adaptive array processing for wideband nulling in GPS systems. Conference Record of the ThirtySecond Asilomar Conference on Signals, Systems \& Computers, 1998, 2: 1132-1336.

[6] Kreng, J.; Sue, M.; Sieu Do; Krikorian, Y.; Raghavan, S., "Telemetry, Tracking, and Command Link Performance Using the USB/STDN Waveform," Aerospace Conference, 2007 IEEE, vol., no., pp.1,15, 3-10 March 2007

[7] Liu Suxiao; Xiong Huagang; Feng Wengquan; Zhao Hongbo, "A New Subcarrier Demodulator of Satellite Telemetry Approaching to the Ideality Based on the Digital Signal," Future Information Technology and Management Engineering, 2009. FITME '09. Second International Conference on, vol., no., pp.191,194, 13-14 Dec. 2009

[8] Oleski, P.J.; Patton, R.W.; Bharj, S.S.; Thaduri, M., "Transmit receive module for space ground link subsystem (SGLS) and unified S-band (USB) satellite telemetry, tracking and commanding (TT and C), and communications," Military Communications Conference, 2004. MILCOM 2004. 2004 IEEE, vol.2, no., pp.880,885 Vol. 2, 31 Oct.-3 Nov. 2004

[9] Jianhong Xiang, Lili Guo, Qingling Liu. Study of GPS Nulling Antenna Based on Space-Time Processing Algorithm. 5th International Conference on Wireless Communications, Networking and Mobile Computing, 2009. WiCom '09, 2009, 1-4.

[10] Yong Wang and Yihua Hu. 2012. Performance of UWB Satellite Communication System under Narrowband Interference. In Proceedings of the 2012 International Conference on Electronics, Communications and Control (ICECC '12). IEEE Computer Society, Washington, DC, USA, 1917-1919. 
[11] Wei Liu, "Adaptive Broadband Beamforming with Spatial-Only Information," Digital Signal Processing, 2007 15th International Conference on, vol., no., pp.575,578, 1-4 July

[12] X. Huang and Y. J. Guo (2011) Frequency-Domain AoA Estimation and Beamforming with Wideband Hybrid Arrays, IEEE Transactions on Wireless Communications 10(8): 2543-2553, doi: 10.1109/TWC.2011.062211.100439.

[13] E. L. Hixson and K.T. Au (1970); Widebandwidth Constant Beamwidth Acoustic Array, J. Acoust. Soc. Am. 48(1): 117 doi: http://dx.doi.org/10.1121/1.1974937.

[14] Smith, R. P. (1970)Constant Beamwidth Receiving Arrays for Broad Band Sonar Systems, Acta Acustica united with Acustica, 23( 1): 21-26.

[15] S. Shirvani Moghaddam, M.R. Pishgoo (2013) New Ideas to Improve the Performance of Frequency Invariant Wideband Antenna Array Beamforming," Majlesi Journal of Telecommunication Devices, Vol. 2, No. 4, pp. 135140, Dec.2013.

[16] S. Shirvani Moghaddam, N. Solgi (2011) A Comparative Study on TDL and SDL Structures for Wideband Antenna Array Beamforming, International Journal on Communications Antenna and Propagation (IRECAP), 1(4): 388-395.

[17] S. S. Moghaddam and N. Solgi (2012) New approach on TDL-based wideband antenna beamforming for radio sources close to the endfire, 20th Iranian Conference on Electrical Engineering (ICEE2012), Tehran pp. 1108-1113. doi: 10.1109/IranianCEE.2012.6292520

[18] Van Trees H L. Optimum Array Processing. New York, USA: John Wiley\& Sons Inc, 2002.

[19] Guo Q L, Sun C. Time-domain nearfield wideband beamforming based on fractional delay filters. The 3rd International Conference on Communication Software and Networks (ICCSN), Xi' an, China, May 27-29, 2011.

[20] Duan H P, Ng B P, See C M S, et al. Broadband beamforming using TDL-form IIR filters. IEEE Transactions on Signal Processing, 2011, 55(3): 990-1002.

[21] Kuts Y, Scherbak L, Sokolovska G.. Methods of processing broadband and narrowband radar signals. Microwaves, Radar and Remote Sensing Symposium(MRRS), Kiev, Ukraine, Aug 25-27, 2011.

[22] Marek Blok and Maciej Sac. 2014. Variable Fractional Delay Filter Design Using a Symmetric Window. Circuits Syst. Signal Process. 33, 10 (October 2014), 3223-3250.

[23] Hauser, Helwig; Groller, Eduard; Theussl, Thomas, "Mastering Windows: Improving Reconstruction," Volume Visualization, 2000. VV 2000. IEEE Symposium on, vol., no., pp.101,108, 9-10 Oct. 2000

[24] M. Blok, Farrow Structure Implementation of Fractional Delay Filter Optimal in Chebyshev sense vol. 6159, in Proc. SPIE, p. 61594K (2005).

[25] Wenxuan Yao; Zhaosheng Teng; Qiu Tang; Peili Zuo, "Adaptive Dolph-Chebyshev window-based S transform in time-frequency analysis," Signal Processing, IET, vol.8, no.9, pp.927,937, 122014

[26] B. Ramesh Reddy, Dr. A.Subbarami Reddy, Dr. P.Chandrashekar Reddy. The Effect of Shape Parameter " $\alpha$ ” in Dolph-Chebyshev Window on the SNR Improvement of MST RADAR Signals. International Journal of Electronics \& Communication Technology, March 2012, 3(1): 7-11.

[27] Subhadeep Chakraborty . Design and Realization of Digital FIR Filter using Dolph-Chebysheb Window. International Journal of Computer Science \& Engineering Technology (IJCSET), Jul 2013, 4(7): 987-996

[28] B.D. Van Veen, K.M. Buckley, Beamforming: a versatile approach to spatial filtering, IEEE Acoustics, Speech, and Signal Processing Magazine, April 1988, 5(2): 4-24.

[29] E.W. Vook, R.T. Compton Jr., Bandwidth performance of linear adaptive arrays with tapped delay-line processing, IEEE Transactions on Aerospace and Electronic Systems, July1992, 28(3): 901-908.

[30] L. Yu, N. Lin, W. Liu, R. Langley, Bandwidth performance of linearly constrained minimum variance beamformers, in: Proceedings of the IEEE International Workshop on Antenna Technology, Cambridge, UK, March2007, pp.327-330.

[31] Liu W, Weiss S. Wideband beamforming concept and techniques. United Kingdom: John Wiley \& Sons Ltd. 2010: 19-60. 